\title{
Non-HDL cholesterol and LDL cholesterol in the dyslipidemic classification in patients with nonalcoholic fatty liver disease
}

\author{
Tingting Du', Xingxing Sun ${ }^{2}$ and Xuefeng $\mathrm{Yu}^{1 *}$
}

\begin{abstract}
Background: Low-density lipoprotein cholesterol (LDL-C) always underestimates the true cholesterol burden in patients with nonalcoholic fatty liver disease (NAFLD). We aimed to compare LDL-C and non-high-density lipoprotein cholesterol (non-HDL-C) in the identification of high-risk dyslipidemic phenotypes in those with NAFLD.

Methods: We conducted a cross-sectional analysis using a cohort of 9560 apparently healthy Chinese adults who underwent comprehensive health checkups including abdominal ultrasonography.

Results: Among 3709 patients with NAFLD, the prevalence of abnormal LDL using LDL-C was 68.5\%, whereas the prevalence was relatively lower when using non-HDL-C (55.9\%). The concordance between non-HDL-C- and LDL-Cbased diagnoses of abnormal LDL was similar in the hypertriglyceridemic $(\kappa=0.56 ; 95 \% \mathrm{Cl} 0.52-0.60)$ and normotriglyceridemic subgroups $(\kappa=0.47 ; 95 \% \mathrm{Cl} 0.44-0.51$ ). Non-HDL-C detected fewer patients with abnormal LDL than LDL-C in normotriglyceridemic patients. However, non-HDL-C detected more patients with abnormal LDL than LDL-C in hypertriglyceridemic patients: 114 of the 1662 patients considered as abnormal LDL according to LDL-C fell into the normonon-HDL-C phenotype, whereas 204 of the 1662 patients considered as abnormal LDL according to non-HDL-C fell into the normoLDL-C phenotype.
\end{abstract}

Conclusion: Among patients with NAFLD, LDL-C is superior to non-HDL-C in the detection of high-risk phenotypes in normotriglyceridemic patients, whereas non-HDL-C seems to be superior in hypertriglyceridemic patients.

Keywords: Nonalcoholic fatty liver disease, LDL-cholesterol, Non-HDL-cholesterol

\section{Background}

The worldwide prevalence of nonalcoholic fatty liver disease (NAFLD) is increasing rapidly, affecting between $15 \%-40 \%$ of adults $[1,2]$. Dyslipidemia that frequently coexist with NAFLD [3] has been identified as a major modifiable risk factor for the accelerated development of cardiovascular disease (CVD) [4]. Although no licensed pharmacological lipid-lowering strategy in patients with NAFLD exists, it is widely accepted that the lipidlowering strategies for NAFLD and CVD are similar, aimed primarily at reducing low-density lipoprotein cholesterol (LDL-C) $[5,6]$. Nevertheless, the major features of dyslipidemia in patients with NAFLD are an

\footnotetext{
* Correspondence: xuefengyu188@gmail.com

'Department of Endocrinology, Tongji Hospital, Tongji Medical College, Huazhong University of Science and Technology, Wuhan 430030, China Full list of author information is available at the end of the article
}

atherogenic lipid profile, consisting of high triglyceride (TG) levels, low high-density lipoprotein cholesterol (HDL-C), and an increase in TG-rich lipoproteins (including very-low-density lipoprotein [VLDL] and intermediate-density lipoprotein [IDL]), and small dense LDL particles) [3]. LDL-C concentrations have generally been reported to be at normal levels in the setting of NAFLD [7, 8]. Thus, LDL-C underestimates the true cholesterol burden in NAFLD as its concentrations do not fully capture the whole mass of lipoprotein particles [7]. Non-high-density lipoprotein cholesterol (non-HDLC) represents a composite measure that encompasses the total cholesterol content within all VLDL, IDL and small dense LDL particles. A growing body of evidence has highlighted that non-HDL-C levels are at least moderately increased in NAFLD [8-10]. Furthermore, a recent study has demonstrated that non-HDL-C is 
stronger than other lipoproteins in predicting the onset of NAFLD [9]. In addition, emerging data have indicated that non-HDL-C is a better predictor of CVD than LDL$C[11,12]$. Therefore, measuring non-HDL-C may better identify lipid abnormalities in patients with NAFLD. However, no comparison has been made between nonHDL-C and LDL-C in the classification of patients with NAFLD into dyslipidemic phenotypes. Hence, we aimed to compare the classification of a group of patients with NAFLD into dyslipidemic phenotypes using non-HDL-C and LDL-C.

\section{Methods}

\section{Study population}

The study participants were Chinese employees and retired workers aged 20-100 years from the Wuhan Iron and Steel Company (WISCO), which is one of the largest iron and steel companies in China. Full details of the study have been described elsewhere [13]. The present cohort included employees and retired workers free of known CVD who received a comprehensive health examination (including abdominal ultrasonography) at the Healthcare system, WISCO general Hospital, between June 2008 and December $2010(n=15,199)$.

All subjects were asked to complete a standard questionnaire that gathered information on alcohol consumption habits, histories of current and previous illness, and medical treatments. We excluded 5639 participants from this study, comprising 1271 with alcohol consumption in amounts $>70 \mathrm{~g} /$ week for women (73) and >140 g/week for men (1198), 857 participants with hepatitis B surface antigen (HBsAg) positivity, and 1467 missing information on age, sex, anthropometric assessment, lipid measurements, test results for HBsAg, or liver ultrasound scans. In addition, to avoid the effects of lipid-lowering on all lipid parameters, 933 participants with lipid-lowering medication use were excluded. Furthermore, 1111 individuals with diabetes (defined using the 2015 American Diabetes Association (ADA) criteria [14] of a fasting plasma glucose $\geq 126 \mathrm{mg} / \mathrm{dl}$ or taking anti-diabetic medications for diabetes) were also excluded as diabetes has a strong independent relationship with increased levels of TG, decreased levels of HDL-C, and increased risk of NAFLD. The remaining available 9560 participants (6022 men and 3538 women) were included in our data analysis (Fig. 1). The fact that men accounted for $63 \%$ of total participants was in consistent with the proportion of male employees at WISCO. According to the Private Information Protection Law, information that might identify subjects was safeguarded by the Health Examination Center. This study was approved by the institutional review board of WISCO general Hospital. Because we only retrospectively accessed a de-identified database for purposes of analysis, informed consent requirement was exempted by the institutional review board. The procedures followed were in accordance with the ethical standards of the responsible

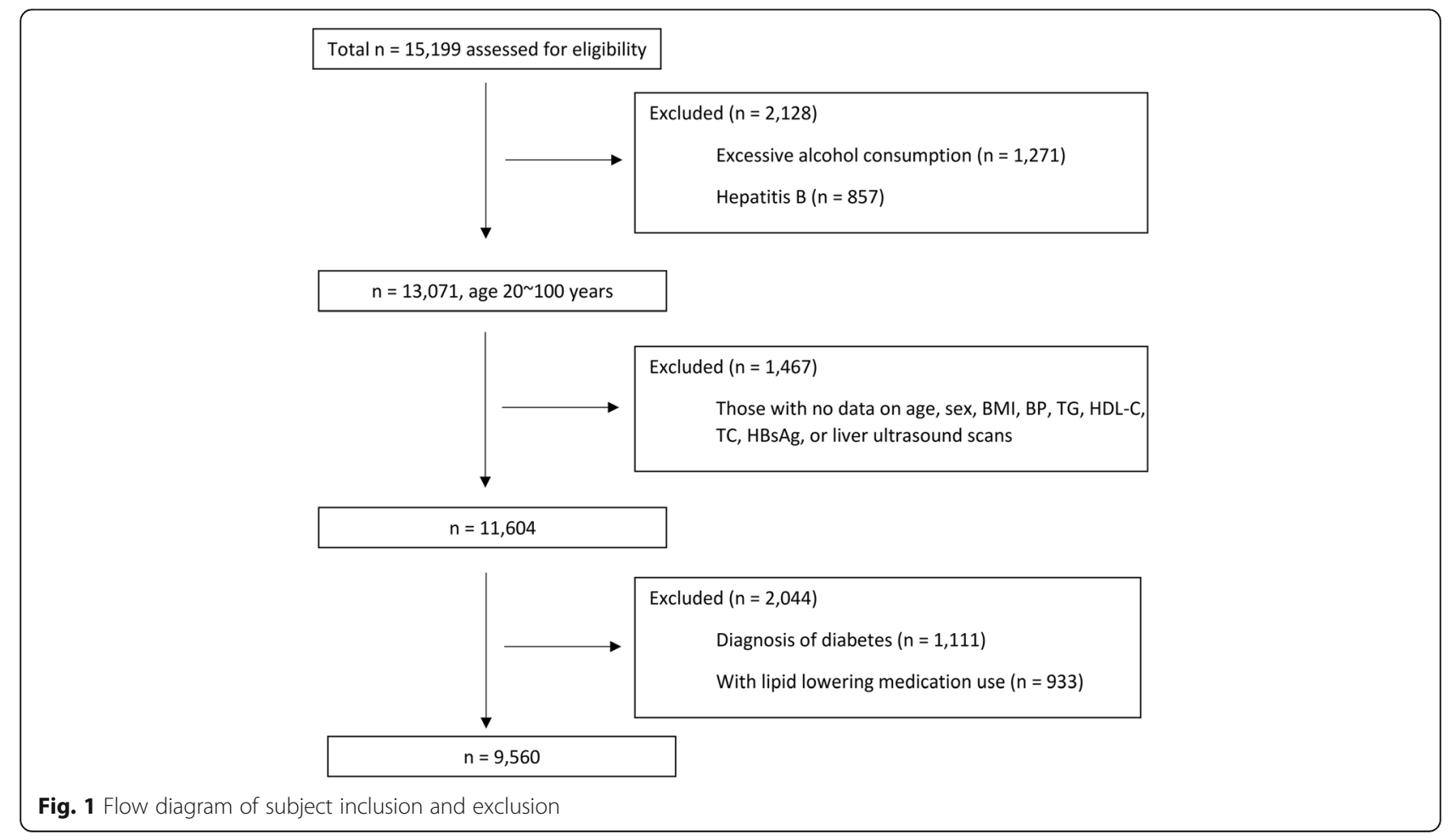


committee on human experimentation and with the Helsinki declaration of 1975, as revised in 1983.

\section{Anthropometric and biochemical measurements}

Anthropometric measurements, including weight, height, and systolic/diastolic blood pressure (BP) were measured following standardized protocols from the World Health Organization (WHO). Body mass index (BMI) was calculated as weight (in kilograms) divided by the square of height (in meters). Participants' seated BP was measured twice for every $5 \mathrm{~min}$ on the right arm after $5 \mathrm{~min}$ of rest by trained nurses with a sphygmomanometer. The mean of the two readings was used in data analysis.

Overnight fasting (at least $8 \mathrm{~h}$ ) blood samples were collected from the antecubital vein of each individual. Biochemical measurements, including assessment of fasting plasma glucose, total cholesterol (TC), TG, LDLC, HDL-C, alanine aminotransferase, uric acid, and hepatitis viral antigen/antibody, were measured enzymatically on an autoanalyzer (Hitachi 7600, Ltd., Tokyo, Japan). Non-HDL-C was calculated as TC minus HDLC. All the blood measurements were followed the same protocol.

\section{Assessment of NAFLD}

Ultrasound tests were performed by trained sonographers using a high-resolution, real-time scanner (model SSD-2000; Aloka Co., Ltd., Tokyo Japan). One experienced radiologists used standard criteria in evaluating the images for the presence or absence of hepatic fat [15]. Generally, the diagnoses of fatty liver was based on the presence of stronger echoes in the hepatic parenchyma compared with echoes in the kidney or spleen parenchyma [16].

\section{Definitions}

According to the current Adult Treatment Panel III of the National Cholesterol Education Program (NECP/ ATP III) guidelines [17], elevated TG is defined as $\geq 150 \mathrm{mg} / \mathrm{dl}$.

According to the ADA and the American College of Cardiology Foundation (ACC) report [18], elevated non$\mathrm{HDL}-\mathrm{C}$ is defined as $\geq 130 \mathrm{mg} / \mathrm{dl}$.

\section{Statistical analysis}

All statistical analyses were performed using SPSS software (version 12.0 for windows; SPSS, Chicago, IL, USA). Continuous variables were presented as medians and interquartile ranges (IQR) due to their skewed distribution. Categorical variables were presented as percentages. Kruskal-Wallis analysis of median test was used followed by the Mann-Whitney $U$ test for pairwise comparisons. Bonferroni correction was applied to adjust $P$-values for multiple comparisons. Chi-square test was performed to assess differences in proportions across groups. Of the 9560 participants studied, 3709 patients were identified as NAFLD. The NAFLD patients were divided into four mutually exclusive groups by the presence or absence of TG $\geq 150 \mathrm{mg} / \mathrm{dl}$ and non-HDL$\mathrm{C} \geq 130 \mathrm{mg} / \mathrm{dl}$. For comparison, patients were also categorized by the conventional approach based on TG and LDL-C cut points. For these analyses, levels of $150 \mathrm{mg} /$ $\mathrm{dl}$ for TG and $100 \mathrm{mg} / \mathrm{dl}$ for LDL-C were chosen. LDL$\mathrm{C}$ values corresponding to non-HDL-C concentrations are not available, a value of $100 \mathrm{mg} / \mathrm{dl}$ for LDL-C was chosen for identification of patients as dyslipidemic phenotypes per consensus report from the ADA/ACC panel [18]. Spearman correlation was adopted to assess coefficients between non-HDL-C and LDL-C. The kappa (к) statistic was calculated to test for an agreement between non-HDL-C- and LDL-C-based identification of dyslipidemic phenotypes. Values for $\mathrm{K}$ value can be between 0 and 1 , with a value of $\geq 0.75$ signifies perfect agreement, whereas with a value of $<0.40$ indicating poor agreement. Venn diagram was constructed as a visual display of concordance/discordance among TG-, LDL-C and non-HDLC-based classification of participants. Significance was accepted at a two-tailed $p<0.05$.

\section{Results}

Characteristics of subjects with and without NAFLD were described elsewhere [19]. Using the conventional classification, $16.0 \%$ were identified as normal, $15.5 \%$ as hypertriglyceride-normoLDL-C, $39.2 \%$ as normoTG-increased LDL-C, and $29.3 \%$ as hypertriglyceride-increased LDL-C (Fig. 2a). Hence, $68.5 \%$ had abnormal LDL, as evidenced by increased

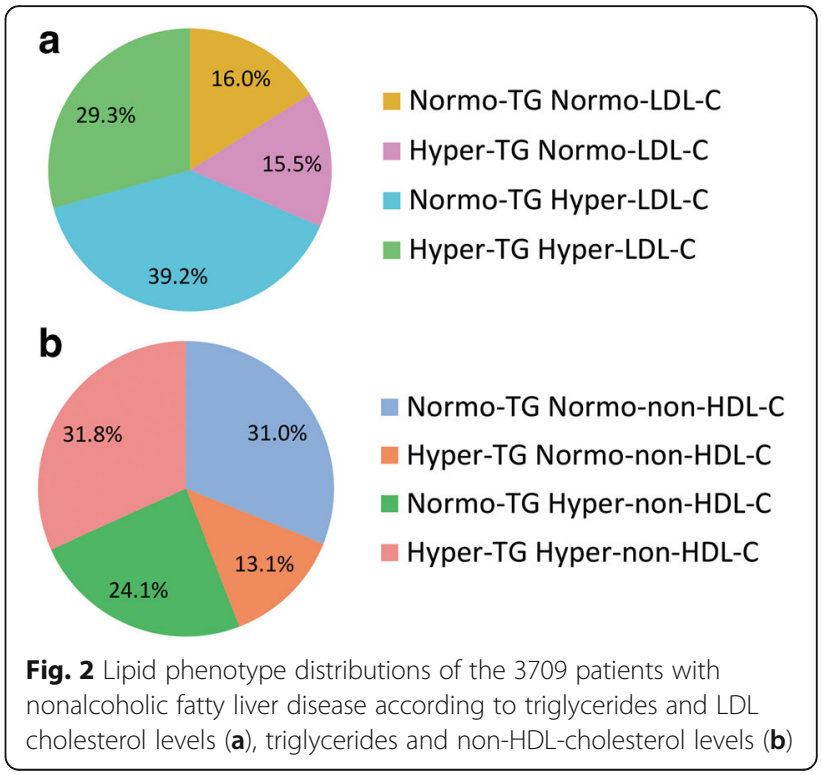


LDL-C. When using TG and non-HDL-C to identify dyslipidemic phenotypes, $31.0 \%$ were identified as normal, $13.1 \%$ as hypertriglyceridemic-normononHDL-C, $24.1 \%$ as normoTG-hyper-non-HDL-C, and $31.8 \%$ as hypertriglyceridemic-hyper-non-HDL-C. (Fig. 2b). In total, $55.9 \%$ of the patients with NAFLD had abnormal LDL particle number and therefore abnormal LDL, as evidenced by increased non-HDL-C.

The characteristics of the four groups in Fig. 2a were showed in Table 1. Individuals with dislipidemia, irrespective of hypertriglyceridemia or increased LDL-C or by both, had higher levels of systolic BP, and manifested more worse lipid profile. Table 2 showed the characteristics of the four groups in Fig. 2b. Individuals with dislipidemia, irrespective of hypertriglyceridemia or increased non-HDL-C or by both, had higher levels of systolic BP and alanine aminotransferase, and manifested more worse lipid profile.

When using non-HDL-C and LDL-C to identify patients with abnormal LDL, discordant classifications occurred for $18.3 \%$ of participants who had an LDL-C $\geq$ $100 \mathrm{mg} / \mathrm{dl}$ and non-HDL-C $<130 \mathrm{mg} / \mathrm{dl}$, and for $5.6 \%$ who had an non-HDL-C $\geq 130 \mathrm{mg} / \mathrm{dl}$ and $\mathrm{LDL}-\mathrm{C}<$ $100 \mathrm{mg} / \mathrm{dl}$.

The correlations of non-HDL-C with LDL-C according to NAFLD status and TG levels were displayed in Table 3 . In the NAFLD state, the correlation between non-HDL-C and LDL-C is similar in the hypertriglyceridemic $(r=0.561, P<0.01)$ and normotriglyceridemic subgroups $(r=0.553, \mathrm{P}<0.01)$. We then evaluated the discordance between classifications based on LDL-C and non-HDL-C according to TG levels $(\mathrm{TG}<150 \mathrm{mg} / \mathrm{dl}$ and $\mathrm{TG} \geq 150 \mathrm{mg} / \mathrm{dl})$
(Fig. 3). The concordance between non-HDL-C- and LDL-C-based diagnoses of abnormal LDL was moderate in both the hypertriglyceridemic ( $=0.56 ; 95 \%$ CI $0.52-0.60)$ and normotriglyceridemic ( $=0.47$; 95\% CI 0.44-0.51) subgroups. Non-HDL-C detected fewer patients with abnormal LDL than LDL-C in normotriglyceridemic patients: 563 of the 2047 patients considered as abnormal LDL according to LDL$\mathrm{C}$ fell into the normonon-HDL-C phenotype, whereas only 5 of the 2047 patients considered as abnormal LDL according to non-HDL-C fell into the normoLDL-C phenotype (Fig. 3). However, non-HDL$\mathrm{C}$ detected more patients with abnormal LDL than LDL-C in hypertriglyceridemic patients: 114 of the 1662 patients considered as abnormal LDL according to LDL-C fell into the normonon-HDL-C phenotype, whereas 204 of the 1662 patients considered as abnormal LDL according to non-HDL-C fell into the normoLDL-C phenotype.

\section{Discussion}

To our knowledge, this is the first study to report a comparison between LDL-C and non-HDL-C for the classification of patients with NAFLD into dyslipidemic phenotypes.

We noted that the prevalence of discordance defined according to LDL-C and non-HDL-C cut points was common, reaching $23.9 \%$. Although fewer proportions of abnormal LDL were identified by non-HDL-C, nonHDL-C identified high-risk phenotypes that are not detected by standard lipid profile in hypertriglyceridemic patients, indicating that non-HDL-C identifies patients

Table 1 Characteristics of participants by triglycerides (TG) and LDL-cholesterol status

\begin{tabular}{|c|c|c|c|c|}
\hline & Normal-TG normal-LDL-C & Hyper-TG normal-LDL-C & Normal-TG hyper-LDL-C & Hyper-TG hyper-LDL-C \\
\hline$n$ & 595 & 574 & 1452 & 1088 \\
\hline Age (years) & $49.0(41.0-56.0)$ & $53.0(45.0-61.0)^{*}$ & $51.0(42.0-58.0)^{*+}$ & $49.0(41.0-58.0)^{\ddagger}$ \\
\hline Body mass index $\left(\mathrm{kg} / \mathrm{m}^{2}\right)$ & $25.5(24.1-27.4)$ & $25.5(24.0-27.3)$ & $26.0(24.3-27.7)$ & $25.1(23.5-27.0)^{\ddagger}$ \\
\hline Systolic blood pressure (mmHg) & $120.0(114.0-131.0)$ & $127.0(120.0-138.0)^{*}$ & $130.0(120.0-140.0)^{*}$ & $129.0(120.0-138.0)^{*}$ \\
\hline Diastolic blood pressure $(\mathrm{mmHg})$ & $80.0(75.0-90.0)$ & $80.0(74.0-90.0)$ & $80.0(76.0-90.0)$ & $80.0(70.0-85.0)$ \\
\hline Fasting plasma glucose(mmol/l) & $5.2(4.8-5.6)$ & $5.2(4.8-5.6)$ & $5.2(4.8-5.6)$ & $5.1(4.7-5.5)$ \\
\hline Uric acid (mmol/l) & $5.4(4.6-6.3)$ & $6.0(5.3-6.9)^{*}$ & $5.9(5.1-6.8)^{*}$ & $5.4(4.6-6.2)^{\dagger \ddagger}$ \\
\hline Alanine aminotransferase $(\mathrm{U} / \mathrm{L})$ & $22.0(16.0-32.0)$ & $24.0(17.0-33.0)$ & $29.0(20.0-43.0)^{*+}$ & $29.0(20.0-40.0)^{*+}$ \\
\hline Total cholesterol (mg/dl) & $148.3(135.5-158.3)$ & $187.6(173.0-206.9)^{*}$ & $204.4(186.1-226.6)^{*}{ }^{*}$ & $164.9(148.6-183.0)^{*}+\neq$ \\
\hline Triglycerides (mg/dl) & $93.8(67.3-117.7)$ & $107.1(85.0-126.5)^{*}$ & $202.7(173.5-254.4)^{*+}$ & $252.2(194.7-372.6)^{*}+\neq$ \\
\hline LDL-cholesterol (mg/dl) & $83.4(71.0-91.9)$ & $123.6(113.1-140.2)^{*}$ & $127.4(113.1-146.3)^{*}$ & $87.6(77.6-95.0)^{* * \neq}$ \\
\hline HDL-cholesterol (mg/dl) & $48.6(42.1-56.4)$ & $50.6(45.2-57.5)^{*}$ & $45.8(40.9-52.1)^{*}$ & $39.4(34.4-44.4)^{*+\neq}$ \\
\hline Non-HDL-cholesterol (mg/dl) & $97.7(88.4-106.2)$ & $135.1(123.2-151.0)^{*}$ & $157.5(140.7-178.8)^{*}+$ & $121.6(108.1-138.2)^{*+\ddagger}$ \\
\hline
\end{tabular}

Data are medians (interquartile range)

${ }^{*} P<0.008$ compared with individuals with normal-TG normal-LDL-C

${ }^{+} P<0.008$ compared with individuals with hyper-TG normal-LDL-C

${ }^{\ddagger} P<0.008$ compared with individuals with normal-TG hyper-LDL-C 
Table 2 Characteristics of participants by triglycerides (TG) and non-HDL-cholesterol status

\begin{tabular}{|c|c|c|c|c|}
\hline & $\begin{array}{l}\text { Normal-TG normal-non- } \\
\text { HDL-C }\end{array}$ & $\begin{array}{l}\text { Hyper-TG normal- non- } \\
\text { HDL-C }\end{array}$ & $\begin{array}{l}\text { Normal-TG hyper- non- } \\
\text { HDL-C }\end{array}$ & $\begin{array}{l}\text { Hyper-TG hyper- non- } \\
\text { HDL-C }\end{array}$ \\
\hline$n$ & 1153 & 484 & 894 & 1178 \\
\hline Age (years) & $48.0(40.0-55.0)$ & $54.0(46.0-63.0)^{*}$ & $51.0(42.0-58.0)^{*}$ & $50.0(41.0-59.0)^{\dagger}$ \\
\hline Body mass index $\left(\mathrm{kg} / \mathrm{m}^{2}\right)$ & $25.5(24.1-27.2)$ & $25.6(24.1-27.5)$ & $26.0(24.3-27.7)$ & $25.2(23.7-27.1)^{\ddagger}$ \\
\hline $\begin{array}{l}\text { Systolic blood pressure } \\
(\mathrm{mmHg})\end{array}$ & $122.0(117.0-134.0)$ & $128.0(120.0-138.0)^{*}$ & $130.0(120.0-140.0)^{*}$ & $123.0(116.0-135.0)^{\dagger \ddagger}$ \\
\hline $\begin{array}{l}\text { Diastolic blood pressure } \\
(\mathrm{mmHg})\end{array}$ & $80.0(74.0-90.0)$ & $80.0(75.0-90.0)$ & $80.0(77.0-90.0)$ & $80.0(70.0-87.0)$ \\
\hline $\begin{array}{l}\text { Fasting plasma glucose } \\
(\mathrm{mmol} / \mathrm{l})\end{array}$ & $5.1(4.8-5.5)$ & $5.2(4.8-5.6)$ & $5.2(4.8-5.7)$ & $5.1(4.7-5.5)$ \\
\hline Uric acid (mmol/l) & $5.3(4.6-6.3)$ & $5.9(5.2-6.7)^{*}$ & $5.9(5.2-6.9)^{*}$ & $5.4(4.6-6.2)^{\dagger \neq}$ \\
\hline Alanine aminotransferase $(U / L)$ & $22.0(16.0-32.0)$ & $25.0(18.0-35.0)^{*}$ & $29.0(20.0-43.0)^{*}$ & $29.0(20.0-41.0)^{*+}$ \\
\hline Total cholesterol (mg/dl) & $158.3(145.2-167.2)$ & $201.2(188.4-217.4)^{*}$ & $205.4(188.0-227.8)^{*}$ & $159.8(147.1-171.0)^{* t \ddagger}$ \\
\hline Triglycerides (mg/dl) & $94.7(70.8-117.7)$ & $112.4(92.0-130.1)^{*}$ & $221.2(181.4-292.9)^{*+}$ & $202.7(172.6-255.8)^{*}+\neq$ \\
\hline LDL-cholesterol (mg/dl) & $88.0(74.5-99.6)$ & $135.5(124.3-149.4)^{*}$ & $124.7(107.3-145.2)^{*+}$ & $99.6(87.3-111.6)^{*+\ddagger}$ \\
\hline HDL-cholesterol (mg/dl) & $40.5(35.5-45.6)$ & $51.4(45.2-59.1)^{*}$ & $45.2(39.8-51.4)^{* \dagger}$ & $49.0(43.2-56.4)^{*+\neq}$ \\
\hline Non-HDL-cholesterol (mg/dl) & $110.2(96.9-120.5)$ & $146.7(137.1-162.5)^{*}$ & $158.9(143.6-180.3)^{*+}$ & $117.8(106.2-123.2)^{*}+\ddagger$ \\
\hline
\end{tabular}

Data are medians (interquartile range)

${ }^{*} P<0.008$ compared with individuals with normal-TG normal-non-HDL-C

${ }^{+} P<0.008$ compared with individuals with hyper-TG normal-non-HDL-C

${ }^{\ddagger} P<0.008$ compared with individuals with normal-TG hyper-non-HDL-C

at risk better than LDL-C in hypertriglyceridemic patients.

Although LDL-C and non-HDL-C are closely correlated, they assess different elements of lipid metabolism. LDL-C is the amount of cholesterol contained in LDL particles, whereas non-HDL-C is the total amount of cholesterol carried by LDL, IDL, and VLDL particles. Mechanistically, the cholesterol content within LDL particles can vary substantially as cholesterol ester within LDL particles can be exchanged for triglyceride molecules within VLDL particles [20]. The considerable discordance between LDL-C- and non-HDL-C-based identification of dyslipidemia phenotypes supported this notion. In the hypertriglyceridemic state, a triglyceride molecule from VLDL particles is exchanged for a cholesterol ester in LDL particles, producing relatively triglyceride-enriched LDL particles and relatively cholesterol-enriched VLDL particles [20]. Hence, LDL-C underestimates the concentrations of non-HDL-C in the setting of hypertriglyceridemia.

It is already established that hypertriglyceridemia, which was typically observed in NAFLD, accounted for

Table 3 Correlations between non-HDL-C and LDL-C according to nonalcoholic fatty liver disease (NAFLD) status and triglycerides levels

\begin{tabular}{lll}
\hline & Without NAFLD & NAFLD \\
\hline Normotriglyceridemia & 0.554 & 0.553 \\
Hypertriglyceridemia & 0.616 & 0.561 \\
\hline
\end{tabular}

the hepatic over-production of VLDL particles and the increased VLDL size [21]. The increased VLDL particles and VLDL size prevent lipoprotein lipase-mediated clearance of triglyceride molecules within VLDL, thereby producing triglyceride-rich lipoprotein remnants. Hepatic lipases can hydrolyze these remnant particles, producing small dense lipoprotein particles and imparting increased CVD risk, suggesting that normal LDL-C but hyper- non-HDL-C generally reflects increased concentrations of smaller, cholesterol-depleted LDL particles among those with hypertriglyceridemia. All these support the notion that the dysregulation of cholesterol played a vital role in the pathogenesis of NAFLD $[8,22]$.

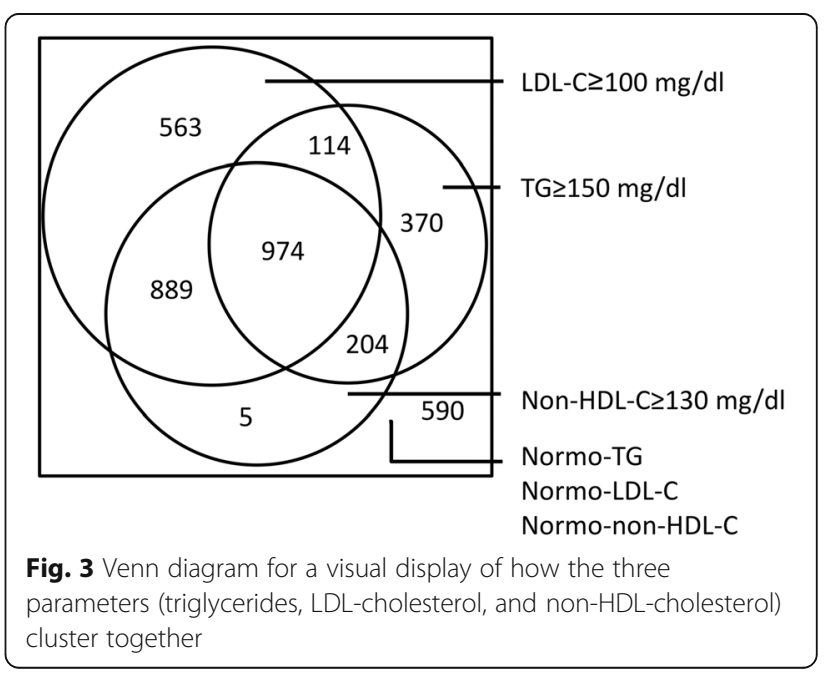


A recent prospective study observed that the increased non-HDL-C levels precedes the onset of NAFLD [9], which further highlighted a causal relationship between the impairment of cholesterol regulation and the NAFLD onset.

In the present study, non-HDL-C identifies a subgroup of patients with normoLDL-C who had hyper-non-HDL$\mathrm{C}$. The identification of this subgroup is noteworthy as the core lipid composition of LDL is altered in a proatherogenic direction. Multiple mechanistic, observational and experimental trials have shown that alterations in VLDL, LDL and IDL synthesis and release may play a role in the pathogenesis of NAFLD [23] and explain the observed increased CVD risk among those with NAFLD. Emerging evidence revealed that cholesterol-lowering therapy was effective in reducing CVD events and improving liver damage among those with NAFLD $[4,24]$. Hence, detection and treatment of dyslipidemia by incorporating non-HDL-C is therefore of importance among patients with NAFLD and for preventing and treating CVD, especially in hypertriglyceridemic patients. Furthermore, accumulating prospective studies indicate the superiority of non-HDL-C over LDL-C in predicting CVD [11, 12]. Our present data supports the currently applicable guidelines that recommend non-HDL-C as alternative targets of therapy to LDL-C for the management of dyslipidemias in individuals with hypertriglyceridemia $[18,25]$.

The present study has several limitations. First, lipoproteins were not measured by the more sophisticated method nuclear magnetic resonance spectroscopy. However, increasing evidence suggested that the association of coronary artery calcification with nuclear magnetic resonance-measured lipoproteins was comparable to that with standard lipids [26]. Second, we studied a cohort of Chinese patients with NAFLD, thus, the present results may not be generalizable to other racial or ethnic patients. Third, the cross-sectional nature of this study makes it difficult to infer causality between different lipid phenotypes and the relative CVD risk among patients with NAFLD. Nevertheless, the analysis of the dyslipidemic classification based on LDL-related measures was not influenced by this particular design. At last, NAFLD was diagnosed by ultrasonography, which is a reasonably accurate technique for detecting modest amounts of liver fat ( $>30 \%$ liver fat in filtration), participants with minor amounts of fatty infiltration might not have been captured.

In conclusion, among patients with NAFLD, LDL-C is superior to non-HDL-C in the detection of high-risk phenotypes in normotriglyceridemic patients, whereas non-HDL-C seems to be superior in hypertriglyceridemic patients. Our findings together with the logistical advantages of non-HDL-C (a cost-free test, and can provide an important value in CVD risk stratification) may support it as a first-line component to be evaluated in dyslipidemic classification and for diagnostic and even therapeutic purposes among those with NAFLD in the setting of hypertriglyceridemia.

\section{Abbreviations \\ ACC: American College of Cardiology Foundation; ADA: American Diabetes Association; BMI: body mass index; BP: blood pressure; CVD: cardiovascular disease; HDL-C: high-density lipoprotein cholesterol; IDL: intermediate-density lipoprotein; IQR: interquartile range; LDL-C: low-density lipoprotein-cholesterol; NAFLD: nonalcoholic fatty liver disease; non-HDL-C: non-high-density lipoprotein cholesterol; TC: total cholesterol; TG: triglyceride; VLDL: very-low- density lipoprotein; WHO: World Health Organization; WISCO: Wuhan Iron and Steel Company}

\section{Acknowledgements}

The authors thank all study participants for their cooperation.

Funding

None

Availability of data and materials

The data are available from the corresponding author upon reasonable request.

\section{Authors' contributions}

TTD conceived the study design, wrote the first draft of the manuscript, analyzed the data, contributed to interpretation of results, commented on drafts, and approved the final version. XXS contributed to interpretation of results, commented on drafts, and approved the final version. XFY is the guarantor of this work, and had full access to all the data in the study and takes responsibility for the integrity of the data and the accuracy of the data analysis.

\section{Ethics approval and consent to participate}

This study was approved by the institutional review board of WISCO general Hospital. Because we only retrospectively accessed a de-identified database for purposes of analysis, informed consent requirement was exempted by the institutional review board. All procedures followed were performed in accordance with the ethical standards of the responsible committee on human experimentation and with the 1975 Helsinki Declaration and its later amendments.

\section{Consent for publication}

Not applicable

\section{Competing interests}

The authors declare that they have no competing interest.

\section{Publisher's Note}

Springer Nature remains neutral with regard to jurisdictional claims in published maps and institutional affiliations.

\section{Author details}

${ }^{1}$ Department of Endocrinology, Tongji Hospital, Tongji Medical College, Huazhong University of Science and Technology, Wuhan 430030, China. ${ }^{2}$ Department of Anesthesiology, Tongji Hospital, Tongji Medical College, Huazhong University of Science and Technology, Wuhan 430030, China.

Received: 6 June 2017 Accepted: 22 November 2017

Published online: 02 December 2017

\section{References}

1. Browning JD, Szczepaniak LS, Dobbins R, Nuremberg P, Horton JD, Cohen $\mathrm{JC}$, et al. Prevalence of hepatic steatosis in an urban population in the United States: impact of ethnicity. Hepatology. 2004;40(6):1387-95. 
2. Vernon G, Baranova A, Younossi ZM. Systematic review: the epidemiology and natural history of non-alcoholic fatty liver disease and non-alcoholic steatohepatitis in adults. Aliment Pharmacol Ther. 2011;34(3):274-85.

3. Speliotes EK, Massaro JM, Hoffmann U, Vasan RS, Meigs JB, Sahani DV, et al. Fatty liver is associated with dyslipidemia and dysglycemia independent of visceral fat: the Framingham heart study. Hepatology. 2010;51(6):1979-87.

4. Athyros VG, Tziomalos K, Gossios TD, Griva T, Anagnostis P, Kargiotis K, et al. Safety and efficacy of long-term statin treatment for cardiovascular events in patients with coronary heart disease and abnormal liver tests in the Greek Atorvastatin and coronary heart disease evaluation (GREACE) study: a post-hoc analysis. Lancet. 2010;376(9756):1916-22.

5. American Gastroenterological Association. American Gastroenterological Association medical position statement: nonalcoholic fatty liver disease. Gastroenterology. 2002;123(5):1702-4

6. Athyros VG, Mikhailidis DP, Didangelos TP, Giouleme Ol, Liberopoulos EN, Karagiannis A, et al. Effect of multifactorial treatment on non-alcoholic fatty liver disease in metabolic syndrome: a randomised study. Curr Med Res Opin. 2006;22(5):873-83.

7. Choi SH, Ginsberg HN. Increased very low density lipoprotein (VLDL) secretion, hepatic steatosis, and insulin resistance. Trends Endocrinol Metab. 2011;22(9):353-63.

8. DeFilippis AP, Blaha MJ, Martin SS, Reed RM, Jones SR, Nasir K, et al. Nonalcoholic fatty liver disease and serum lipoproteins: the multi-ethnic study of atherosclerosis. Atherosclerosis. 2013;227(2):429-36.

9. Zelber-Sagi S, Salomone F, Yeshua H, Lotan R, Webb M, Halpern Z, et al. Non-high-density lipoprotein cholesterol independently predicts new onset of non-alcoholic fatty liver disease. Liver Int. 2014;34(6):e128-35.

10. Corey KE, Lai M, Gelrud LG, Misdraji J, Barlow LL, Zheng H, et al. Non-highdensity lipoprotein cholesterol as a biomarker for nonalcoholic steatohepatitis. Clin Gastroenterol Hepatol. 2012;10(6):651-6.

11. Boekholdt SM, Arsenault BJ, Mora S, Pedersen TR, LaRosa JC, Nestel PJ, et al. Association of LDL cholesterol, non-HDL cholesterol, and apolipoprotein B levels with risk of cardiovascular events among patients treated with statins: a meta-analysis. JAMA. 2012;307(12):1302-9.

12. Thanassoulis G, Williams K, Ye K, Brook R, Couture P, Lawler PR, et al. Relations of change in plasma levels of $L D L-C$, non-HDL-C and apoB with risk reduction from statin therapy: a meta-analysis of randomized trials. J Am Heart Assoc. 2014;3(2):e000759.

13. Du T, Yu X, Yuan G, Zhang J, Sun X. Combined influence of nonalcoholic fatty liver and body size phenotypes on diabetes risk. Cardiovasc Diabetol. 2015;14144.

14. American Diabetes Association. (2) classification and diagnosis of diabetes. Diabetes Care. 2015;38(SupplS8-S16).

15. Mittelstaedt C, Vincent $L$. The urinary tract. Abdominal ultrasound. 1st ed. New York: Churchill Livingston; 1987. p. 269.

16. Saadeh S, Younossi ZM, Remer EM, Gramlich T, Ong JP, Hurley M, et al. The utility of radiological imaging in nonalcoholic fatty liver disease. Gastroenterology. 2002;123(3):745-50.

17. Expert Panel on Detection Evaluation, And Treat ment of High Blood Cholesterol in Adults. Executive summary of the third report of the National Cholesterol Education Program (NCEP) expert panel on detection, evaluation, and treatment of high blood cholesterol in adults (adult treatment panel III). JAMA. 2001;285(19):2486-97.

18. Brunzell JD, Davidson M, Furberg CD, Goldberg RB, Howard BV, Stein $\mathrm{JH}$, et al. Lipoprotein management in patients with cardiometabolic risk: consensus statement from the American Diabetes Association and the American College of Cardiology Foundation. Diabetes Care. 2008; 31(4):811-22

19. Du T, Sun X, Yuan G, Zhou X, Lu H, Lin X, et al. Sex differences in the impact of nonalcoholic fatty liver disease on cardiovascular risk factors. Nutr Metab Cardiovasc Dis. 2017;27(1):63-9.

20. Sniderman AD, Scantlebury T, Cianflone K. Hypertriglyceridemic hyperapob: the unappreciated atherogenic dyslipoproteinemia in type 2 diabetes mellitus. Ann Intern Med. 2001;135(6):447-59.

21. Cohen DE, Fisher EA. Lipoprotein metabolism, dyslipidemia, and nonalcoholic fatty liver disease. Semin Liver Dis. 2013;33(4):380-8.

22. Simonen P, Kotronen A, Hallikainen M, Sevastianova K, Makkonen J, Hakkarainen A, et al. Cholesterol synthesis is increased and absorption decreased in non-alcoholic fatty liver disease independent of obesity. J Hepatol. 2011:54(1):153-9.
23. Fujita K, Nozaki $Y$, Wada K, Yoneda M, Fujimoto $Y$, Fujitake $M$, et al. Dysfunctional very-low-density lipoprotein synthesis and release is a key factor in nonalcoholic steatohepatitis pathogenesis. Hepatology. 2009;50(3): 772-80.

24. Kimura Y, Hyogo H, Yamagishi S, Takeuchi M, Ishitobi T, Nabeshima Y, et al. Atorvastatin decreases serum levels of advanced glycation endproducts (AGEs) in nonalcoholic steatohepatitis (NASH) patients with dyslipidemia: clinical usefulness of AGEs as a biomarker for the attenuation of NASH. J Gastroenterol. 2010;45(7):750-7.

25. European Association for Cardiovascular Prevention and Rehabilitation, Reiner Z, Catapano AL, De Backer G, Graham I, et al. ESC/EAS guidelines for the management of dyslipidaemias: the task force for the management of dyslipidaemias of the European Society of Cardiology (ESC) and the European atherosclerosis society (EAS). Eur Heart J. 2011;32(14):1769-818.

26. Hisamatsu T, Fujiyoshi A, Miura K, Ohkubo T, Kadota A, Kadowaki S, et al. Lipoprotein particle profiles compared with standard lipids in association with coronary artery calcification in the general Japanese population. Atherosclerosis. 2014;236(2):237-43.

\section{Submit your next manuscript to BioMed Central and we will help you at every step:}

- We accept pre-submission inquiries

- Our selector tool helps you to find the most relevant journal

- We provide round the clock customer support

- Convenient online submission

- Thorough peer review

- Inclusion in PubMed and all major indexing services

- Maximum visibility for your research

Submit your manuscript at www.biomedcentral.com/submit
) Biomed Central 